\title{
Achondrogenesis type 1B
}

\author{
Andrea Superti-Furga
}

\section{Historical notes}

In 1952, the name achondrogenesis (Greek for "not producing cartilage") was given by Marco Fraccaro, a young Italian pathologist (later to become a well known cytogeneticist), to the condition he observed in a stillborn female with severe micromelia and marked histological changes of cartilage. ${ }^{1}$ Fraccaro noted a similar case published by Parenti in $1936 .^{2}$ Fraccaro's report (written in Italian) came to the knowledge of Hans Grebe, who in 1939 had observed sisters (aged 7 and 11 years, born to consanguineous parents from the Black Forest region of Germany) with markedly short limbs and digits but normal trunk. Grebe became convinced that his patients were affected by the same achondrogenesis as Fraccaro's short limbed stillborn patient, and he had his report (written in German) published in the same journal. ${ }^{3}$ However, the condition described by Grebe was different (although superficially similar to Fraccaro's achondrogenesis because of limb shortening) and has later become known as Grebe chondrodysplasia or Grebe syndrome.

The name achondrogenesis was then used to characterise the most severe forms of chondrodysplasia in humans, invariably lethal before or shortly after birth. Precise figures on the incidence of achondrogenesis are not available, but it is not exceedingly rare: reviews with large numbers of patients have been published. ${ }^{45}$

In the 1970s, the heterogeneity of achondrogenesis was recognised. Using a combination of radiological and histological criteria, achondrogenesis type I (then also called Fraccaro-Houston-Harris type) and type II (called Langer-Saldino type) were distinguished. ${ }^{67}$ In 1976 , it was found that chondrocytes of some (though not all) type I patients contained cytoplasmic inclusions. ${ }^{8}$ In 1983, a new classification of achondrogenesis (types I to IV) was proposed ${ }^{9}$ and adopted in the McKusick catalogue. This radiological classification,

\section{( $(\mathcal{M}$ Med Genet 1996;33:957-961)}

Key words: achondrogenesis type 1B; cartilage; differential diagnosis. which tried to provide a quantitative basis (the "femoral cylinder index") for qualitative changes, did not prove helpful and was later abandoned. In the late 1980 s, it was shown that achondrogenesis type II was caused by structural mutations in collagen II and thus constituted the severe end of the spectrum of the collagen II chondrodysplasias. ${ }^{10-13}$

Borochowitz et $a l^{14}$ provided convincing histological criteria for the further subdivision of achondrogenesis type I into IA (with apparently normal cartilage matrix but inclusions in chondrocytes) and IB (with abnormal cartilage matrix; see below). These findings were confirmed by another group shortly thereafter. ${ }^{15}$ Using these criteria, some older published cases can be unequivocally diagnosed as type IB, ${ }^{1617}$ others as type IA, ${ }^{18}$ while Parenti's case probably should be classified as type II. ${ }^{214}$ The classification of achondrogenesis type IB as a separate group has recently been confirmed by biochemical and molecular findings (see below). The history of achondrogenesis shows how subjective clinical and radiological classifications can be. Histology and ultrastructure have allowed a more solid classification and provided the basis for biochemical and molecular studies.

The present classification of achondrogenesis is shown in table 1 . Recessive inheritance of achondrogenesis type IA is not formally proven, and this type may still contain different subgroups. To avoid misunderstandings in the era of gene symbols and computerised databases, it is advisable (though regrettable) to convert Roman numerals to Arabic numerals. Accordingly, the following abbreviations are proposed: achondrogenesis type 1A (ACG1A), achondrogenesis type 1B (ACG1B), and achondrogenesis type 2 (ACG2).

\section{Clinical features of achondrogenesis type $1 \mathrm{~B}$}

Newborns with ACG1B frequently present in breech position. After delivery, their appearance is immediately perceived as abnormal

Table 1 Classification of achondrogenesis

\begin{tabular}{lllll}
\hline Name & Former eponyms & Abbreviation & Inheritance & Molecular basis \\
\hline Achondrogenesis type 1A & Houston-Harris type & ACG1A & AR (?) & Unknown \\
Achondrogenesis type 1B & Fraccaro type & ACG1B & AR & Mutations in sulphate transporter gene, DTDST, on 5q \\
Achondrogenesis type 2 & Langer-Saldino type & ACG2 & AD (de novo) & Mutations in collagen II gene, COL2A1, on 12q \\
\hline
\end{tabular}




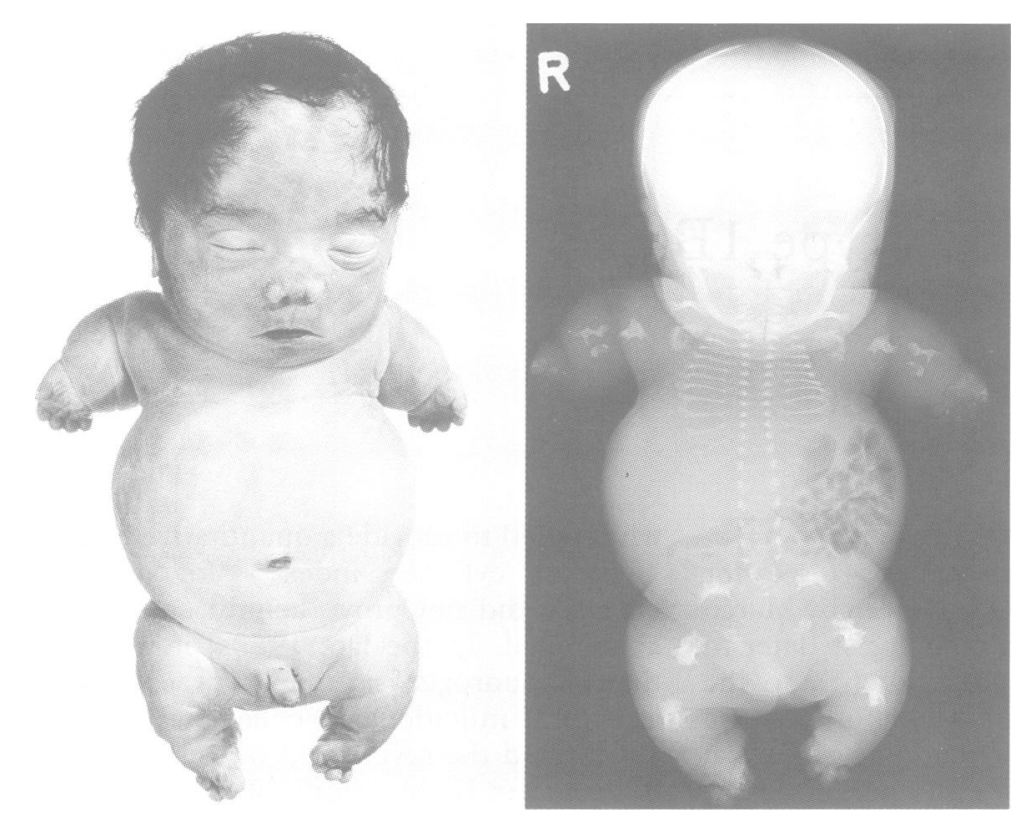

Figure 1 (Left) Clinical appearance of a patient with ACG1B, born at 34 weeks and died 25 minutes after birth. Note the flat face, the narrow thorax with protuberant abdomen, and particularly the severe micromelia with short stubby fingers and toes. (Right) X ray of the same newborn. Following the resuscitation attempts, there is air in the stomach and intestine but not in the lungs. For details of the skeletal system, refer to the text. For radiographs of other newborns with $A C G 1 B$, see refs 14, 15, 16, 17, or 21. (Reprinted from ref 19, with permission of the publisher.)

(fig 1, left). The abundance of soft tissue relative to the short skeleton gives these newborns a fat or hydropic appearance. There is a disproportion between the head, which is of normal or near normal size, and the rest of the body, which is much shorter than normal. The face is flat, the neck short, and the soft tissue of the neck may be thickened. The thorax is narrow and the abdomen is protuberant. Umbilical or inguinal herniae are frequent. The most striking abnormality concerns the limbs. They are severely shortened, sometimes resembling flippers, sometimes, when an articular crease at the hip or shoulder is present, resembling sausages. The fingers and toes are similarly short and stubby. The feet and toes are rotated inwards in a fashion reminiscent of diastrophic dysplasia. The external genital organs are unremarkable. Death may occur before birth for causes which are not understood. Even when heart action is present at birth, respiratory insufficiency follows shortly.

Fetuses with achondrogenesis may be ascertained by the observation of short femora on routine ultrasonography during pregnancy. Other ultrasonographic signs may be nuchal oedema, reduced rump length, poor ossification of the vertebral bodies and of the limb bones (leading to difficulties in determining their length), and polyhydramnios. If pregnancy is interrupted, it is imperative to obtain good clinical and radiographic documentation as well as appropriate biological material to allow a precise diagnosis and adequate counselling (see below).

\section{Radiological features}

Radiological features have been reviewed in six cases with biochemically and molecularly proven ACG1B, ${ }^{141519-22}$ as well as in published cases who could be classified as ACG1B by their histological features. ${ }^{1617}$ The following should be noted: (1) there is a certain degree of variability, and no single feature should be regarded as obligatory; (2) contrary to what has been reported, ${ }^{14}$ distinction between ACG1B and ACG1A on radiographs is not always possible; (3) the degree of ossification is age dependent, and caution is needed in comparing radiographs from patients at different gestational ages.

In ACG1B, the skull is only mildly affected: it is slightly less ossified than expected for gestational age, and the orbits may be extended laterally and superiorly. The disproportion between the skull of almost normal size and the hypoplastic skeleton is noticeable. The clavicles are mildly affected; they are ossified, and, albeit somewhat short, of near normal shape. The scapulae are small and their contour is irregular. The vertebral bodies are usually not ossified; at most, rudimentary calcification in the central part is seen. The vertebral lateral pedicles are usually ossified, and part of the neural arches may also be. The ribs are slightly thinner and much shorter than normal; they are usually not fractured (although occasionally, one or two fractures have been seen) and show slight cupping at their distal ends. The iliac bones are smaller than usual and only their upper (cranial) half is ossified, often in an irregular fashion; their shape can be compared to that of a crescent or a paraglider. The ischium is not ossified or only minimally.

The tubular bones are those most markedly affected (fig 1). Femora and humeri are usually shortened to a degree where no major axis can be recognised. As some metaphyseal spurring occurs, these bones end up resembling cartoon stars, thorn apples, or, for the haematological expert, acanthocytes. The tibiae and fibulae are similarly misshapen and the fibulae are not ossified in more premature cases. In most cases, the ulnae are amorphous, while the radii show some tubulation and may have a peculiar distal dichotomy thereby resembling a waterfork. The carpals and phalanges are usually very poorly ossified and can rarely be identified on whole baby $x$ rays. Sometimes bipartite ossification of the medial phalanges can be seen.

\section{Histology of cartilage}

In ACG1B, the cartilage matrix is rarefied and partially replaced by a larger number of cells. After haematoxylin-eosin staining, the matrix does not have the characteristic homogeneous, ground glass appearance; instead, it shows coarsened collagen fibres. The fibres are often radially arranged around individual chondrocytes and bridge the space between chondrocytes. The fibres are particularly dense around chondrocytes where they can form socalled collagen rings ${ }^{14}$ (fig 2). The coarsened fibres are seen well on silver stained sections. In normal cartilage, cationic dyes such as toluidine blue or Alcian blue give a homogeneous, deep blue, or violet staining of the matrix owing to the abundance of polyanionic sulphated pro- 


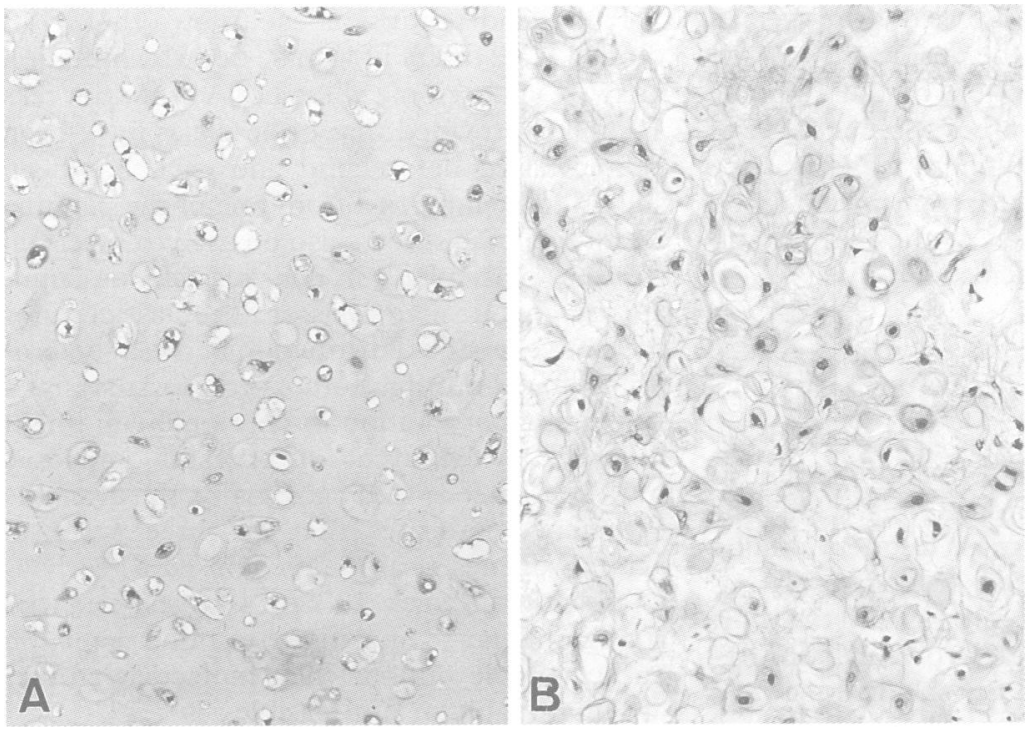

Figure 2 (A) Section through normal fetal cartilage. The cartilage matrix is homogeneous and no structures can be recognised. (B) Section through ACG1B cartilage (case shown in fig 1, see ref 19). The number of cells is increased. The matrix is devoid of ground substance and fibrillar structures can be recognised, which tend to form rings around chondrocytes. (Both parts: haematoxylin-eosin staining.)

teoglycans. In ACG1B cartilage, the intensity of staining with these dyes is very much reduced, and the matrix between the coarsened collagen fibres may not stain at all. These features, probably the result of a deficiency in sulphated proteoglycans, ${ }^{1519}$ distinguish ACG1B both from ACG1A (where the matrix is close to normal and inclusions can be seen in chondrocytes) and from ACG2 (where cationic dyes give a normal staining pattern and coarsening of the fibres and collagen rings is not seen).

\section{Physical and biochemical findings in cartilage}

ACG1B cartilage is brownish and translucent rather than white like normal cartilage, and friable rather than firm and elastic. Its consistency is similar to that of a cooked apple. It can be easily cut through with a scalpel. In this respect, it resembles cartilage from severe collagen II disorders such as ACG2 or Kniest

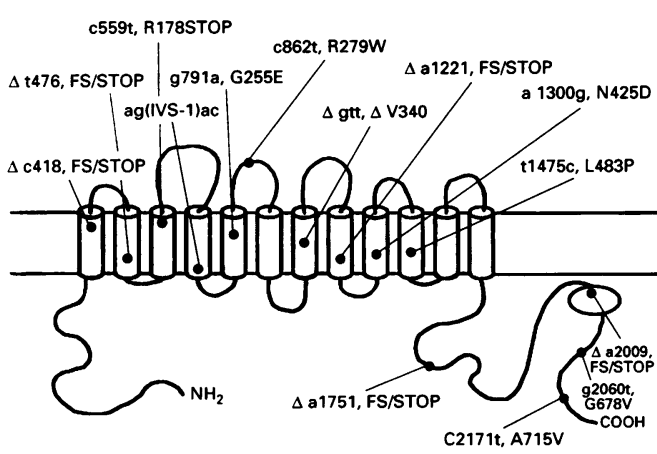

Figure 3 Schematic representation of the diastrophic dysplasia sulphate transporter (modified from ref 23) with localisation of the known pathogenic mutations. Several of these mutations have been found in $A C G 1 B, A O 2$, and DTD; the combination of mutations is responsible for modulating the phenotype. Further details concerning individual mutations can be found in ref 26; nucleotide and amino acid numbering follow ref 23. dysplasia. The structure of collagens II, IX, and XI was shown to be normal in cartilage from the patient shown in fig 1 (B Steinmann, unpublished data, 1988), suggesting to me a defect in some other structural component.

The total sulphate content of ACG1B cartilage is less than one-fifth that of control cartilage. ${ }^{20}$ When cartilage extracts are separated by polyacrylamide or agarose gel electrophoresis and the gels stained with cationic dyes, the sulphated proteoglycans appear to be reduced in concentration ${ }^{19}$ and to migrate more slowly because of reduced negative charge. ${ }^{22}$ Chondrocytes and skin fibroblasts cultured from ACG $1 B$ patients are unable to incorporate exogenous sulphate ${ }^{1922}$; this is best shown by a double labelling test with ${ }^{3} \mathrm{H}$-glycine and ${ }^{35} \mathrm{~S}$ sodium sulphate. ${ }^{20}$ In both cell types, a defect in the uptake of extracellular sulphate can be shown. ${ }^{2022}$

\section{Molecular basis of ACG1B}

The discovery of the sulphate incorporation defect in ACG1B came at the same time as identification of the gene for a sulphate transporter, DTDST, ${ }^{23}$ on chromosome $5 \mathrm{q}$ as the gene locus responsible for the non-lethal disorder, diastrophic dysplasia (DTD), ${ }^{24}$ for which no biochemical clues had existed. This made DTDST a plausible candidate gene for ACG1B. Indeed, a series of mutations in the DTDST gene have been identified in patients with ACG1B, DTD, and a disorder of intermediate severity called atelosteogenesis type 2 (AO2). Mutations include point mutations and deletions leading to premature stop codons, substitutions or deletions of amino acids within transmembrane domains, substitutions of amino acids in intra- or extracellular domains, and a presumed mutation lying outside the coding region but causing low mRNA levels. ${ }^{20232526}$ Genotype-phenotype correlations indicate that the amount of residual activity of the sulphate transporter modulates the phenotype from lethal ACG1B to nonlethal DTD ${ }^{26}$ Homozygosity or compound heterozygosity for mutations predicting stop codons or structural mutations in transmembrane domains are associated with ACG1B, while mutations in extracellular loops or the cytoplasmic tail or the presumed regulatory mutation giving low mRNA usually result in the less severe phenotypes, $\mathrm{AO} 2$ and DTD. Thus, there is a chondrodysplasia family caused by allelic mutations in the DTDST gene. ${ }^{26}$ Heterozygotes are of normal stature and do not appear to have degenerative joint disease, confirming recessive inheritance.

Impaired activity of the sulphate transporter in chondrocytes and fibroblasts results in the synthesis of proteoglycans which are not sulphated or only insufficiently, ${ }^{1922}$ probably by depletion of intracellular sulphate. Proteoglycan undersulphation has a pronounced effect on the composition of the extracellular matrix of cartilage, as shown by the physical, biochemical, and histological findings. ${ }^{1922}$ How this ultimately leads to the chondrodysplasia is not known. Interestingly, there is no clear 
indication that other organs besides the skeletal system are affected by the disorder, in spite of the ubiquitous expression of the sulphate transporter. ${ }^{23}$ Possibly, other tissues have a lower requirement for sulphate which can be met either by transport through other anion carriers or by endocellular synthesis from sulphur containing amino acids.

\section{Differential diagnosis and diagnostic procedures}

The differential diagnosis of achondrogenesis is that of lethal osteochondrodysplasias, a long list of conditions. ${ }^{27}$ The most frequent among these disorders are lethal osteogenesis imperfecta, thanatophoric dysplasia, and the short rib-polydactyly syndromes. In osteogenesis imperfecta, the skull is soft, the sclerae blue, and the bones bowed but clearly not as short as in achondrogenesis. In thanatophoric dysplasia, the limbs are longer and the shape of the thorax is narrow but elongated. The short ribpolydactyly syndromes resemble thanatophoric dysplasia and are usually associated with hexadactyly. Marked limb shortening may suggest Roberts syndrome, in which the axial skeleton is not affected or only mildly. Distinction between ACG1A, ACG1B, and ACG2 on clinical grounds is difficult. Almost normal hands are seen in ACG2, whereas in ACG1A and ACG1B the hands are evidently shortened; radiological evidence of rib fractures may suggest ACG1A.

Most cases with ACG1B occur sporadically and therefore the ultrasonographic or clinical finding of severe short limbed chondrodysplasia arises unexpectedly. Experience shows that in such a setting it is rarely possible to make the correct diagnosis; any diagnosis between osteogenesis imperfecta, thanatophoric dysplasia, and achondrogenesis is a good attempt. However, it is important to secure a later diagnosis by obtaining good radiographs, EDTA blood for DNA analysis, a skin biopsy under sterile conditions for fibroblast culture, and bone and cartilage tissue for histology and biochemistry. The combination of radiological and histological findings will give a provisional diagnosis which can then be confirmed by selected biochemical or molecular investigations or both. For ACG1B, these investigations would include the study of sulphate incorporation in cultured fibroblasts or chondrocytes and mutation analysis of the DTDST gene.

\section{Genetic counselling and prenatal diagnosis}

ACG1B and the related disorders, AO2 and DTD, are inherited as autosomal recessive traits. For a given couple, the recurrence risk is 1 in 4 , or $25 \%$, and thus markedly higher than that for ACG2 which is usually caused by a new dominant mutation. Asymptomatic carriers may be present in the families of affected patients. Therefore, genetic counselling must rely on differentiation between ACG1B and ACG2. This can be done as outlined above.
Prenatal diagnosis or exclusion of ACG1B (and of AO2 or DTD) can be done by mutation analysis of chorionic villus DNA around weeks 10 to 11 , provided that both alleles have been characterised beforehand and each has been shown to be inherited from one of the parents. Mutation analysis can also be used to ascertain carriers, particularly in consanguineous families. Biochemical analysis of fibroblast cultures has not yet enabled distinction between heterozygotes and normal homozygotes. Analysis of sulphate incorporation in chorionic villi might theoretically be used for prenatal diagnosis, but experience is lacking. Finally, the available experience indicates that all three types of achondrogenesis can be recognised as early as in gestational week 13 or 14 by experienced sonographers, ${ }^{28-30}$ making ultrasound detection an acceptable option when molecular studies are unavailable or not feasible.

I am indebted to J Bonaventure, J Briner, D Cohn, G Eich, A Giedion, J Hästbacka, D L Rimoin, A Rossi, Th Stallmach, W Wilcox, B Steinmann, and R Gitzelmann for patient material, laboratory data, financial support, or help in the preparation of the manuscript. This work was supported by the Swiss National the manuscript. This work was supported by the Swiss Nationa tung of the University of Zurich.

1 Fraccaro M. Contributo allo studio del mesenchima osteopoietico - l'acondrogenesi. Folia Hered Pathol (Milano) 1952;1:190-207.

2 Parenti GC. La anosteogenesi. Pathologica (Genova) 1936; 8:447-62

3 Grebe H. Die Achondrogenesis: ein einfachrezessives Erbmerkmal. Folia Hered Pathol (Milano) 1952;2:23-8.

4 Wiedemann HR, Remagen W, Hienz HA, Gorlin RJ, Maroteaux $\mathrm{P}$. Achondrogenesis within the scope of connately manifested generalized skeletal dysplasias. $Z$ Kinderheilkd 1974;116:223-51.

5 Schulte MJ, Lenz W, Vogel M. Letale Achondrogenesis: eine Übersicht über 56 Fälle. Klin Padiatr 1978;191: 327-40.

6 Spranger J, Langer LO, Wiedemann HR. Bone dysplasias an atlas of constitutional disorders of skeletal development. Stuttgart: Gustav Fischer Verlag, 1974.

7 Yang SS, Brough AJ, Garewal GS, Bernstein J. Two types of heritable lethal achondrogenesis. $\mathcal{F}$ Pediatr 1974;85: 796-801.

8 Yang SS, Heidelberger KP, Bernstein J. Intracytoplasmic inclusion bodies in the chondrocytes of type I lethal achondrogenesis. Hum Pathol 1976;7:667-73.

9 Whitley CB, Gorlin RJ. Achondrogenesis: new nosology with evidence of genetic heterogeneity. Radiology 1983; 148:693-8.

10 Eyre DR, Upton MP, Shapiro FD, Wilkinson RH, Vawter $\mathrm{GF}$. Nonexpression of cartilage type II collagen in a case of Langer-Saldino achondrogenesis. Am $\mathcal{f}$ Hum Gene 1986;39:52-67.

11 Godfrey $M$, Hollister DW. Type II achondrogenesishypochondrogenesis: identification of abnormal type II collagen. Am f Hum Genet 1988;43:904-13.

12 Vissing H, D'Alessio M, Lee B, Ramirez F, Godfrey M, Hollister DW. Glycine to serine substitution in the triplehelical domain of pro $\alpha 1$ (II) collagen results in a lethal perinatal form of short-limbed dwarfism. F Biol Chem

13 Spranger J, Winterpacht A, Zabel B. The type II collagenopathies: a spectrum of chondrodysplasias. Eur $\mathcal{f}$ Pediatr 1994;153:56-65

14 Borochowitz Z, Lachman R, Adornian GE, Spear G, Jones $\mathrm{K}$, Rimoin DL. Achondrogenesis type I: delineation of further heterogeneity and identification of two distinct subgroups. $\mathcal{F}$ Pediatr 1988;112:23-31.

15 van der Harten HJ, Brons JTJ, Dijkstra PF, et al. Achondrogenesis-hypochondrogenesis: the spectrum of chondrogenesis imperfecta. Pediatr Pathol 1988;8:571-97.

16 Urso FP, Urso MJ. Achondrogenesis in two sibs. Birth Defects 1974;10:10-17.

17 Nardi F, Gerlini G, Bonucci E. Achondrogenesis: report on a case, with particular reference to ultrastructure and histochemistry. Virchows Arch A Pathol Anat Histol 1974; 363:311-22.

18 Molz G, Spycher MA. Achondrogenesis type I: light and electron-microscopic study. Eur f Pediatr 1980;134:69-74.

19 Superti-Furga A. A defect in the metabolic activation of sulphate in a patient with achondrogenesis type IB. $A m \mathcal{F}$ Hum Genet 1994;55:1137-45.

20 Superti-Furga A, Hästbacka J, Wilcox WR, et al. Achondrogenesis type IB is caused by mutations in the diastrophic dysplasia sulphate transporter gene. Nat Genet 1996;12:100-2.

21 Freisinger P, Stanescu V, Jacob B, Cohen-Solal L Maroteaux J, Bonaventure J. Achondrogenesis type IB 
(Fraccaro): study of collagen in the tissue and in chondrocytes cultured in agarose. Am f Med Genet 1994;49. 439-46.

22 Rossi A, Bonaventure J, Delezoide AL, Cetta G, SupertiFurga A. Undersulphation of proteoglycans synthesized Furga A. Undersulphation of protegglycans synthesized by chondrocytes from a patient with achondrogenesis diastrophic dysplasia sulphate transporter. $\mathcal{F}$ Biol Chem diastrophic dysplasia sulphate transporter. $f$ Biol Chem

23 Hästbacka J, de la Chapelle A, Mahtani MM, et al. The diastrophic dysplasia gene encodes a novel sulphate transporter: positional cloning by fine-structure linkage disequilibrium mapping. Cell 1994;78:1073-87.

24 Lamy M, Maroteaux P. Le nanisme diastrophique. Presse Med 1960;68:977-1980.

25 Hästbacka J, Superti-Furga A, Wilcox WR, Rimoin DI Cohn DH, Lander ES. Atelosteogenesis type II is caused by mutations in the diastrophic dysplasia sulphate trans- porter gene (DTDST): evidence for a phenotypic series involving three chondrodysplasias. Am F Hum Genet 1996; 58:255-62.

26 Superti-Furga A, Rossi A, Steinmann B, Gitzelmann R. A chondrodysplasia family produced by mutations in the diastrophic dysplasia sulphate transporter gene: genotype/ phenotype correlations. Am $\mathcal{F}$ Med Genet 1996;63:144-7.

27 Spranger J, Maroteaux P. The lethal osteochondrodysplasias. Adv Hum Genet 1990;19:1-103.

28 dysplasias. Adv Hum Genet 1990;19:1-103. diagnosis of the skeletal dysplasias. Am f Obstet Gynecol diagnosis of the ste

29 Soothill PW, Vuthiwong C, Rees H. Achondrogenesis type 2 diagnosed by transvaginal ultrasound at 12 weeks' gestation. Prenat Diagn 1993;13:523-8.

30 Meizner I, Barhard Y. Achondrogenesis type I diagnosed by transvaginal ultrasonography at 13 weeks gestation. Am $\mathcal{F}$ Obstet Gynecol 1995;173:1620-2. 